\title{
КОМПЬЮТЕРНОЕ МОДЕЛИРОВАНИЕ ПРОЦЕССОВ, ПРОТЕКАЮЩИХ ПРИ БУРЕНИИ СКВАЖИН
}

\author{
Попова Марина Сергеевна', \\ alleniram83@mail.ru \\ Харитонов Антон Юрьевич², \\ donetskant@yandex.ru

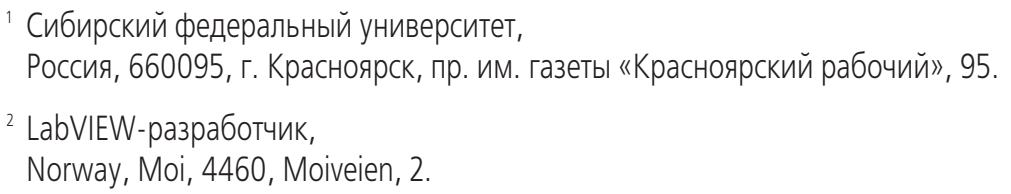

Актуальность. Основным направлением развития бурения скважин является повышение технико-экономических показателей и разработка нового поколения породоразрушающего инструмента, обеспечивающего эффективное разрушение горной породы. Для повышения технико-экономических показателей бурения необходимо изучение процессов, протекающих при бурении скважин. Зачастую они носят сложный характер и нуждаются в детализации в мелких труднодоступных местах призабойной зоны. В некоторых случаях экспериментальные методы исследования затруднительны, требуют материальных затрат или вовсе недоступны. К тому же основным современным требованием к научным исследованиям является комплексный подход, который заключается во всестороннем и одновременном рассмотрении всех процессов, протекающих при бурении скважины. В связи с этим применение компьютерного моделирования является актуальным методом, позволяющим точно, наглядно и достоверно проводить исследования процессов бурения.

Цель: компьютерное моделирование процессов, протекающих при бурении скважин.

объекты: процессы, протекающие при бурении скважин.

Методы: имитационное моделирование, аналитические исследования, анализ.

Результаты. Определены основные процессы бурения, которые необходимо исследовать при проектировании и разработке нового поколения породоразрушающего инструмента. Рассмотрены возможности компьютерного моделирования при исследовании процессов бурения алмазными коронками. На примере упрощенной модели взаимодействия породоразрушающего инструмента с горной породой приведены программные продукты и такие результаты их применения, как толщина слоя породы, снимаемая любым алмазом коронки, координаты местонахождения инструмента в любой момент времени, температура нагрева алмазной однослойной коронки, распределение механических напряжений в породоразрушающем инструменте колонкового бурения. Осуществлено сравнение моделирования одних и тех же процессов бурения с применением различных программных продуктов. Выявлены особенности и точность моделирования процессов бурения. Обосновано использование компьютерного моделирования процессов бурения. Показаны преимущества компьютерного моделирования над экспериментом.

\section{Ключевые слова:}

Компьютерное моделирование, бурение, породоразрушающий инструмент, программирование, параметрическая модель, раскладка алмазов, буровая коронка.

\section{Введение}

В эпоху экономии и борьбы за энергетические ресурсы бурение как способ разведки и добычи полезного ископаемого приобретает решающее значение в развитии мировой экономики. Повышенные требования к технико-экономическим показателям бурения предполагают использование комплексного подхода в научном исследовании буровых процессов [1]. Бурение скважин как модель является сложной системой, состоящей из механических, химических, гидравлических, температурных явлений. Некоторые элементы бурения для достоверности результатов необходимо изучать на микроуровне. Таким образом, исследование процессов бурения является сложной задачей и решение ее привычными методами не всегда остается возможным. Наиболее перспективным в этом направлении является компьютерное моделирование, способное максимально приблизить модель исследования к реальным условиям, сократить время и затраты на разработку инструмента, анализ процессов бурения, визуализировать полученный результат [2].
Современные разработчики бурового инструмента все чаще пользуются общедоступными компьютерными технологиями инженерного проектирования, а также разрабатывают собственные продукты, например, Ideas, Smith Bits с успехом применили компьютерное моделирование в своих разработках. Наиболее популярными в области исследования буровых процессов на сегодняшний день являются CAE (Computer Aided Engineering) системы, основанные на методе конечных элементов, такие как Nastran, Patran, Ansys, позволяющие производить детальное изучение даже в самых мелких элементах и труднодоступных местах расчётной схемы. [3-13]

Основным технико-экономическим показателем бурения является механическая скорость бурения, которая во многом зависит от механизма разрушения горной породы [14-16]. Моделирование движения породоразрушающего инструмента на забое должно включать исследование изменения значения толщины слоя породы, срезаемого различными его резцами; температуры нагрева 
плоскости контакта резцов с породой и влияния ее значения на работу инструмента в целом; линий тока промывочной жидкости; давления жидкости в различных точках скважины и т. д.

\section{Выбор объекта моделирования}

Механическая скорость бурения во многом зависит от качества разрушения горной породы породоразрушающим инструментом. Одним из наиболее эффективных в бурении является алмазный породоразрушающий инструмент. Компьютерное моделирование требует четкой постановки задач и правильной подачи информации для последующей обработки. Поэтому в качестве удобного объекта моделирования выбрана алмазная однослойная коронка. Армирована такая коронка крупными синтетическими монокристаллами с размером зерна около 1,5 мм в диаметре, что позволяет обеспечивать заданный выпуск алмазных резцов [17]. Располагаются монокристаллы на торце коронки по известной радиальной схеме раскладки $[18,19]$. Такое расположение имеет определенный порядок, является закономерным, позволяет присваивать каждому алмазу фиксированные координаты и систематизировать работу линий резания. При выборе в качестве объекта моделирования алмазной однослойной коронки с заданным выпуском крупных синтетических монокристаллов, расположенных по радиальной схеме, важным фактором является симметричность получаемой в результате модели.

\section{О применении компьютерного моделирования при исследовании процессов, протекающих при бурении скважин}

Положение каждого алмазного монокристалла представленной коронки во время работы на забое определяется углом её поворота $(\varphi)$ и углубкой коронки (z) (рис. 1). Каждая линия резания коронки дополнительно характеризуется координатами поверхности забоя $(y(\varphi))$ в каждой своей точке. Толщину слоя породы, срезаемую алмазом в конкретной точке забоя при определенном положении коронки, можно определить из выражения (1) [19]:

$$
h_{i}=z-y_{i},
$$

где $y_{i}$ - координата поверхности забоя перед конкретным алмазом.

При постоянной осевой нагрузке соблюдается условие неизменности площади внедрения всех алмазов в забой, таким образом имеем соотношение (2):

$$
\sum_{i=1}^{n_{a}} h_{i}=n_{\mathrm{K}} h_{a},
$$

где $h_{a}$ - глубина внедрения монокристалла в породу при статическом вдавливании (рис. $1, a)$. Значение $h_{a}$ может рассчитываться по уточненным формулам [20], при этом учитываются свойства разрушаемой горной породы $\left(k_{p}\right)$ и форма алмаза $\left(k_{a}\right)$ [20].

Как показывает аналитическое исследование процесса движения коронки по забою в процессе бурения скважины, при вращении, за счет осевого

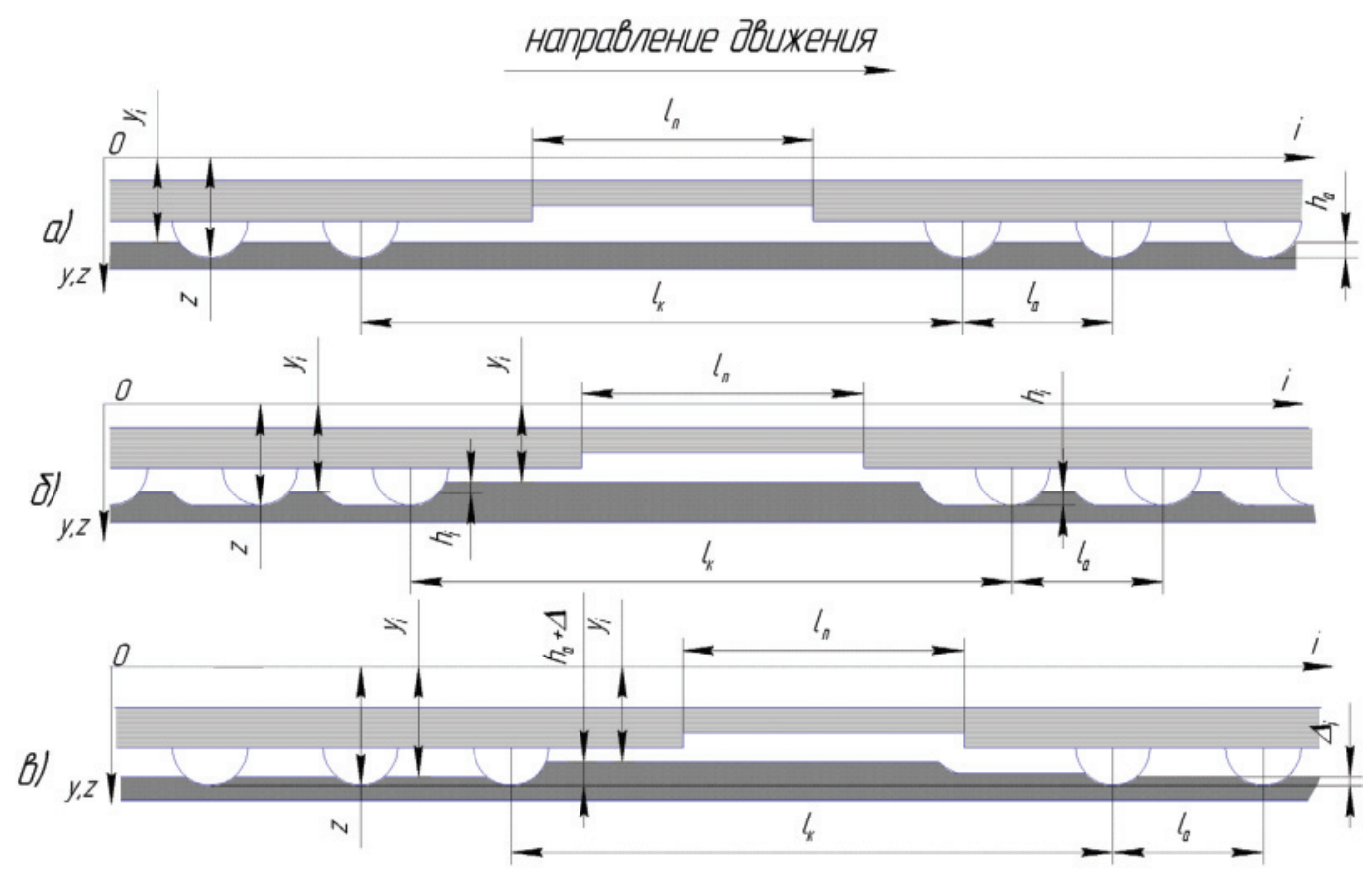

Pис. 1. Схема взаимодействия алмазов коронки одной линии резания с забоем скважины. Углубка алмазов в породу: а) первоначальная; б) при прохождении меньшего расстояния, чел расстояние между алмазами на одной линии резания $\left(l_{\mathrm{a}}\right)$; в) после прохождения расстояния $l_{\text {a }}$

Fig. 1. Diagram of interaction of a single-line crow diamond with a well bottom. Depth of diamonds in the rock: a) initial; б) with the passage of distance smaller than that between the diamonds on the same line of cutting; 8 ) after passing the distance $l_{\mathrm{a}}$ 
усилия, происходит периодическое углубление коронки на величину $\Delta$. Т. е. при каждом акте разрушения породы будет выполняться соотношение:

$$
\sum_{i=1}^{n_{a}}\left(z-y_{i}+\Delta\right)=n_{\mathrm{K}} h_{a},
$$

где $\Delta$ - приращение углубления коронки на каждом акте внедрения в породу.

Из уравнения (3) имеем значение D?, соответствующее определенному положению коронки на забое $[16,18]$.

$$
\Delta=\frac{n_{\mathrm{K}} h_{a}-\sum_{i=1}^{n_{\mathrm{K}}}\left(z-y_{i}\right)}{n_{\mathrm{\kappa}}}=h_{a}-\frac{1}{n_{\mathrm{\kappa}}} \sum_{i=1}^{n_{\mathrm{K}}}\left(z-y_{i}\right) .
$$

Используя полученную математическую модель (4), зная точное расположение синтетических монокристаллов на торце коронки, можно осуществить моделирование процесса бурения алмазной однослойной коронкой и получить значение её периодической углубки $\Delta$, координату нижнего торца коронки $z$ и координаты поверхности забоя $y(\varphi)$ в любой точке и в любой момент времени.

Для реализации такой модели сотрудниками Донецкого национального технического университета разработана программа «koronka2», написанная на языке программирования Delphi. «koronka2» позволяет моделировать процесс движения однослойной алмазной коронки по забою скважины. Программа рассчитывает величину толщины слоя породы, срезаемого каждым алмазом в конкретный момент времени, определяет координату нижнего торца коронки, а также выдает на экран траекторию движение алмазов в осевом направле- нии по мере движения нижнего торца коронки. Данная программа предоставляет возможность при моделировании учитывать характер раскладки алмазов по торцу и конструктивные параметры коронки, что позволяет проводить сравнительную оценку влияния раскладки алмазов как на процесс движения торца коронки, так и на величину нагрузки каждого из алмазов в процессе бурения.

Исходными данными для моделирования являются координаты расположения каждого алмаза в торце коронки, а также величина первоначального углубления алмазов коронки при приложении осевой нагрузки без вращения коронки. Дополнительным необходимым параметром является общее количество точек моделирования, на которое разбивается линия резания.

Однако данный программный продукт имеет ряд недостатков. Одним из которых является зависимость от количества точек, на которые разбивается линия резания коронки. Причем чем больше задано точек в линии резания, тем точнее результаты моделирования. «koronka2» не учитывает свойства породы, профиль резца, режимы бурения.

Один из основных продуктов компании National Instruments - среда графического программирования LabVIEW, за счет возможности выполнения элементов параллельного программирования позволяет устранить некоторые допущения, принятые при использовании программы «koronka2». LabVIEW давно зарекомендовала себя как качественный программный продукт, обеспечивающий полноценные исследования сложных систем. LabVIEW используется в системах сбора и обработ-

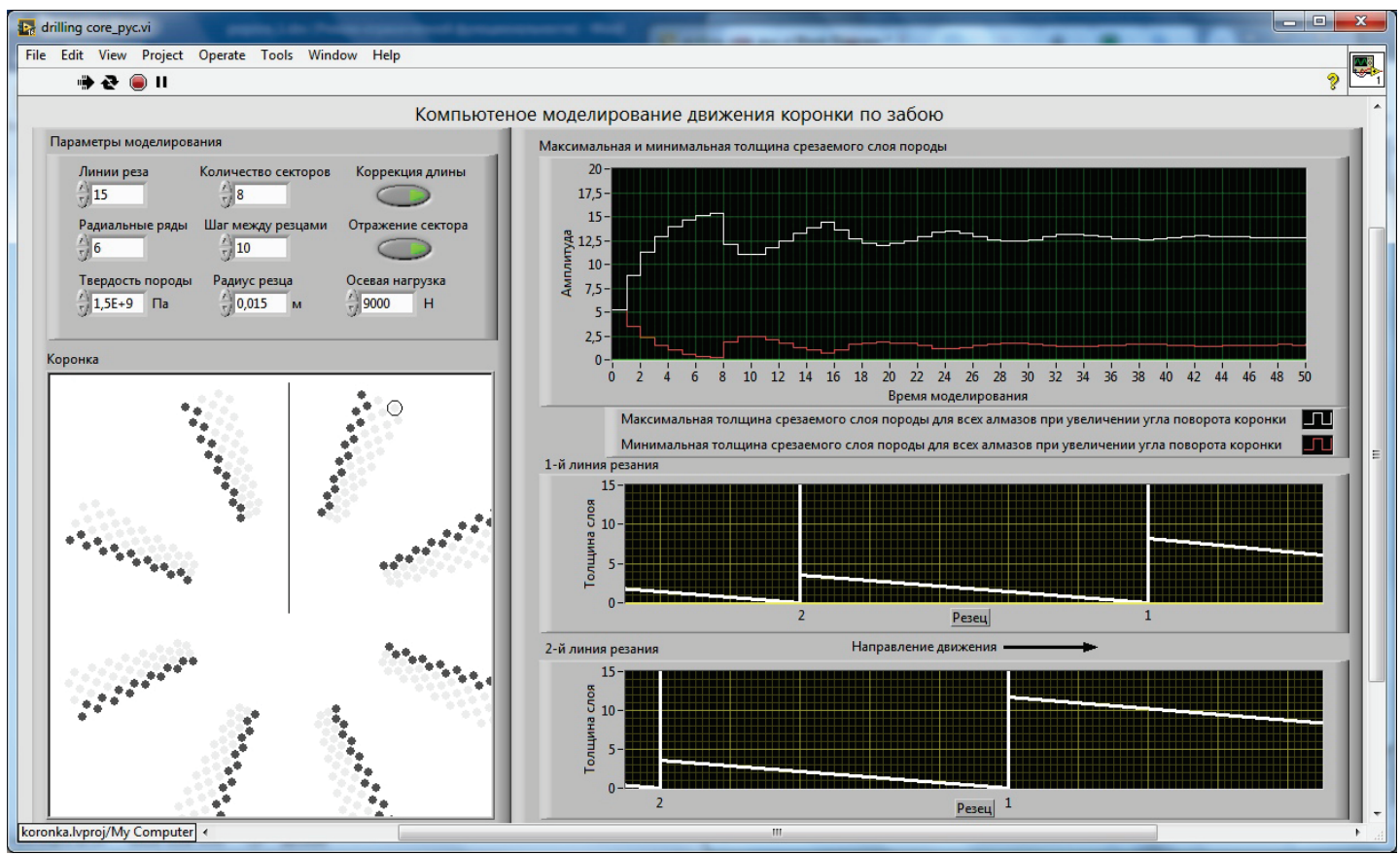

Puс. 2. Интерфейс програмлы моделирования движения аллазной коронки по забою (среда програмлирования LabVIEW)

Fig. 2. Diamond crown motion simulation program interface (LabVIEW programming environment) 

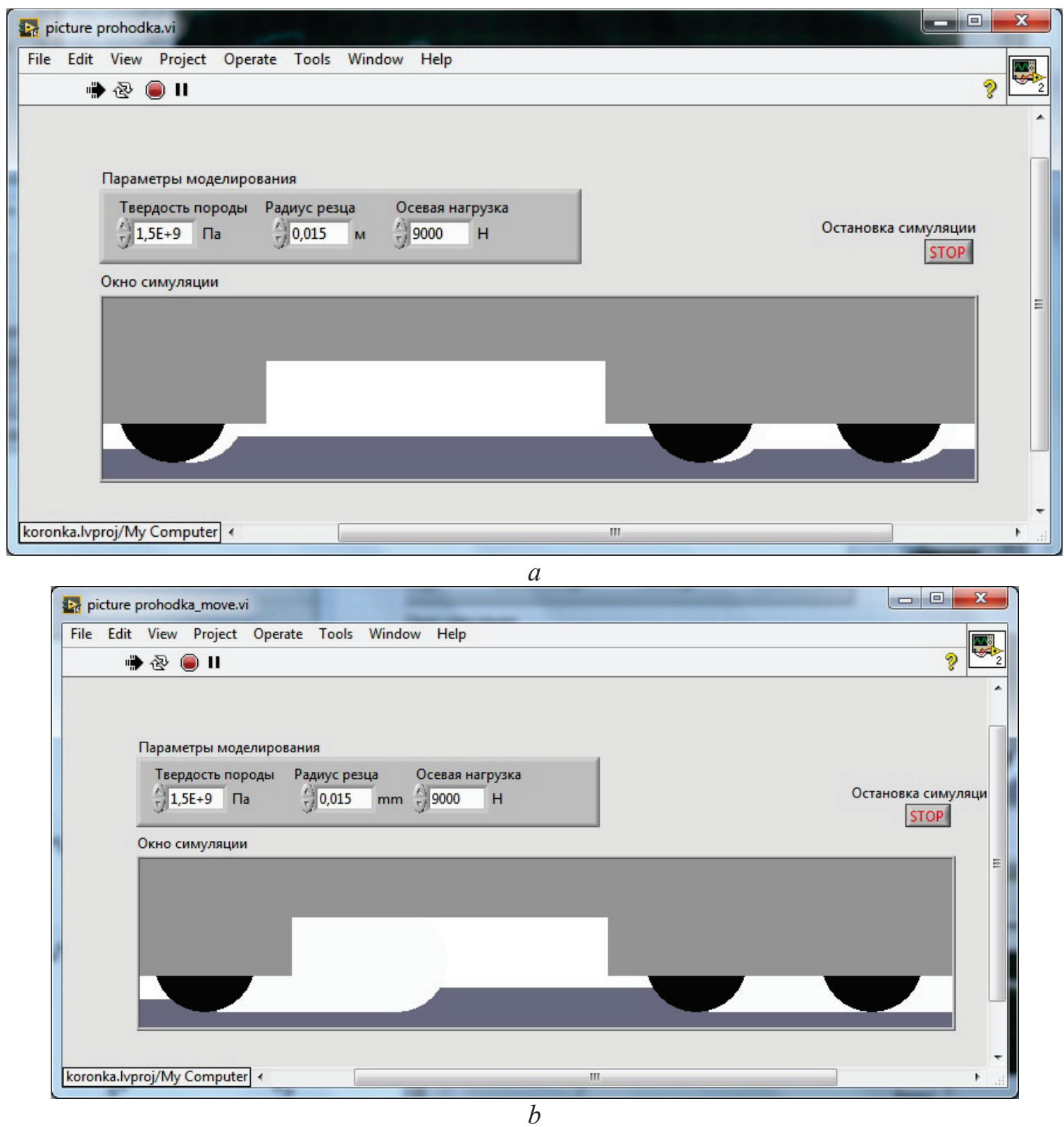

Рис. 3. Моделирование прохода алмазных резиов по породе: а) статическое внедрение коронки в породу; $b$ ) поворот коронки на угол, равный расстоянию между двумя смежными в линии резания алмазами сектора

Fig. 3. Modeling the passage of diamond cutters for the rock: a) static introduction of the crown into the rock; b) rotation of the crown by an angle equal to the distance between two adjacent diamonds in the cutting line

ки данных, а также для управления техническими объектами и технологическими процессами [21]. Идеологически LabVIEW ориентирована на решение задач в области научных исследований [22].

Опираясь на результаты ранее проведенных исследований работы отдельного резца на забое скважины [3], при исследовании эффективности разрушения горной породы породоразрушающим инструментом необходимо учитывать не только толщину слоя породы, срезаемого алмазом, но и остальные элементы сложной системы механизма разрушения породы. За счет принципа параллельного программирования [23] в LabVIEW имеется возможность моделировать поведение всех резцов и влияние резцов друг на друга. Среда графического программирования LabVIEW позволяет создавать дружелюбный графический интерфейс пользователя (рис. 2), характеризующийся гибкой настройкой и удобством конфигурирования [24]. На рис. 1 представлен внешний вид программы, который включает:

1) установку начальных значений программы количество линий, секторов и радиальных рядов резания;

2) окно моделирования расположения резцов коронки; 
3) график результатов моделирования максимальной и минимальной толщины срезаемой породы;

4) график результатов моделирования визуализации двух уровней срезанной породы двумя резцами.

Среда программирования LabVIEW имеет большой набор дополнений и библиотек. Одно из дополнений - работа с графикой, позволяет в режиме реального времени отображать изменение различных геометрических фигур в зависимости от программируемых математических формул [25]. Данное дополнение позволило показать работу коронки с резцами в движении, а также динамику снимаемой резцами породы. На рис. 3 показаны копии экранных снимков моделирования прохода алмазных резцов по породе в двух тактах работы программы.

Реализация на языке LabVIEW является более современной, поскольку опирается на современные средства поддержки научных исследований, что позволит в дальнейшем добавлять новые функции и способы анализа данной научной задачи.

Используя комплексный подход к исследованию буровых процессов (с учетом механических, температурных, гидравлических явлений), протекающих при бурении скважин, часто возникают сложности с построением геометрии имитационной модели. $K$ примеру, при разработке конструкции инструмента, в процессе исследования, неоднократно приходится изменять форму отдельных элементов, их расположение, размеры и т. д. При помощи программного комплекса САПР КОМПАC-3D разработана параметрическая модель, позволяющая осуществлять автоматизированное построение моделей алмазной коронки. При её использовании можно задавать конструктивные параметры коронки: наружный и внутренний диаметр, количество секторов, количество алмазов в секторе, высота сектора, параметры раскладки алмазов и др. Пример полученной геометрии модели приведен на рис. 4.
Для детального изучения распределения механических нагрузок в коронке методом конечных элементов проведено моделирование ее работы на забое скважины с учетом осевых и крутящих усилий. Помимо геометрических данных коронки в качестве исходных в такой системе, как Ansys, возможно использование физико-механических свойств и формы алмаза, способ их закрепления в матрице, состав материала матрицы и режимы бурения. Результаты моделирования хорошо визуализированы (рис. 5) и позволяют сделать вывод о возможности бурения коронками с укороченным до 4 радиальных рядов секторами.

При разработке, особенно алмазного, породоразрушающего инструмента, внимание уделяется температурным процессам, которые могут повлиять на ход проходки скважины. Для исследования температурных процессов на контакте алмазов с горной породой проводится одновременное исследование тепловых и гидравлических явлений, протекающих при бурении скважин. Экспериментальные исследования температурных процессов всячески затруднены и не позволяют осуществлять прямые измерения непосредственно в источнике образования тепла. Моделирование в системе Ansys позволяет производить замер температуры даже внутри тела алмазного резца. При этом система позволяет за основу имитационной модели принять такую часть коронки, которая достоверно учитывает симметрию происходящих процессов, что значительно сокращает ресурсы компьютерного исследования. В этом случае границы модели задаются плоскостями симметрии и находятся в пределах области сектора коронки, проходя через центр промывочного окна.

Составляемая модель (рис. 6) состоит из нескольких областей решения поставленной задачи доменов. Для каждой конструкции коронки составляется своя объемная модель. В каждой модели выделены: домен очистного агента, породы, сектора, корпуса и алмазов.

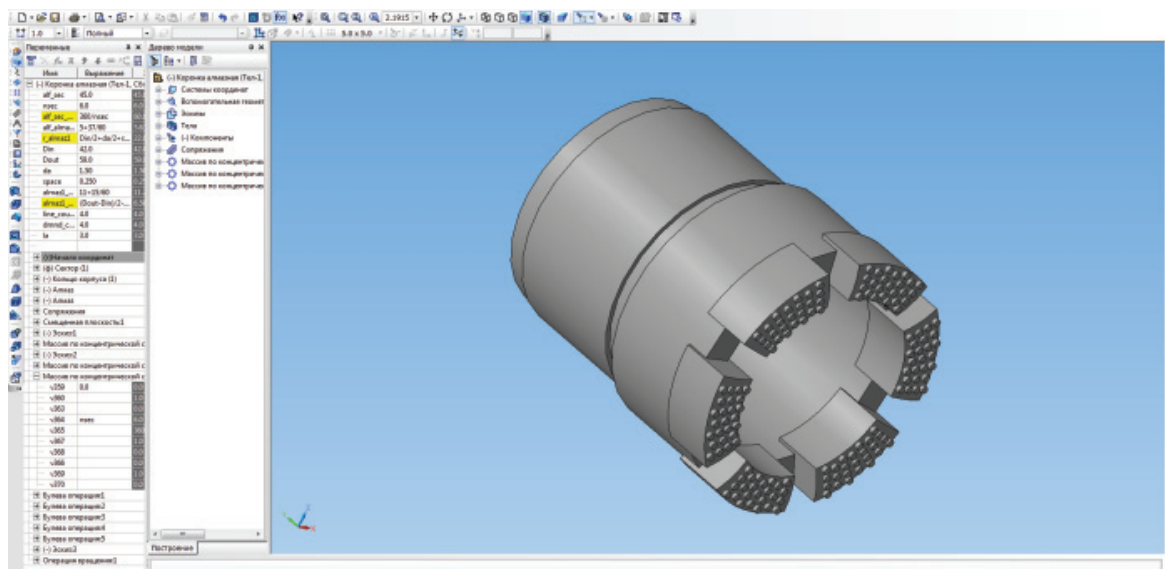

Рис. 4. Пример моделирования конструкиии алмазной однослойной коронки

Fig. 4. Example of modeling the design of a diamond single-layered crown 

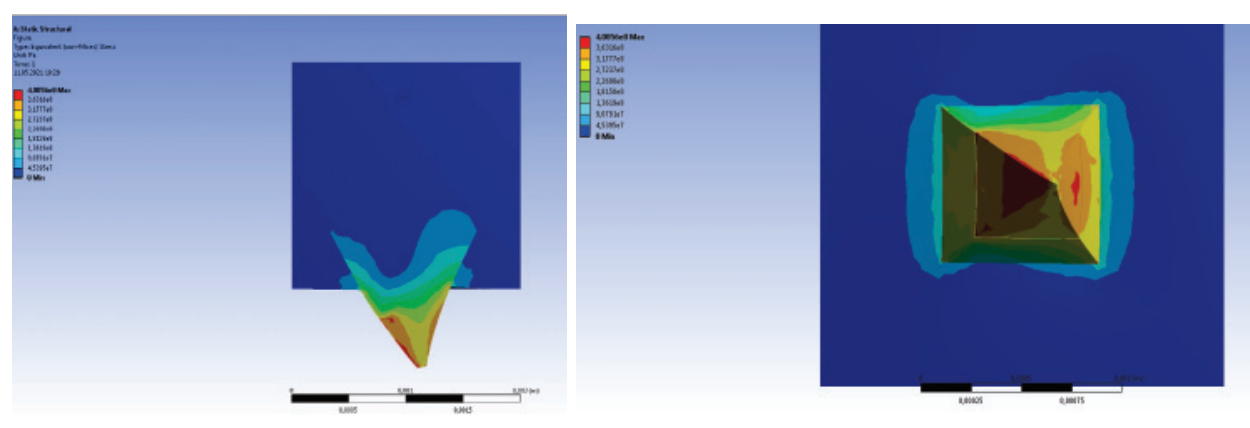

$a$

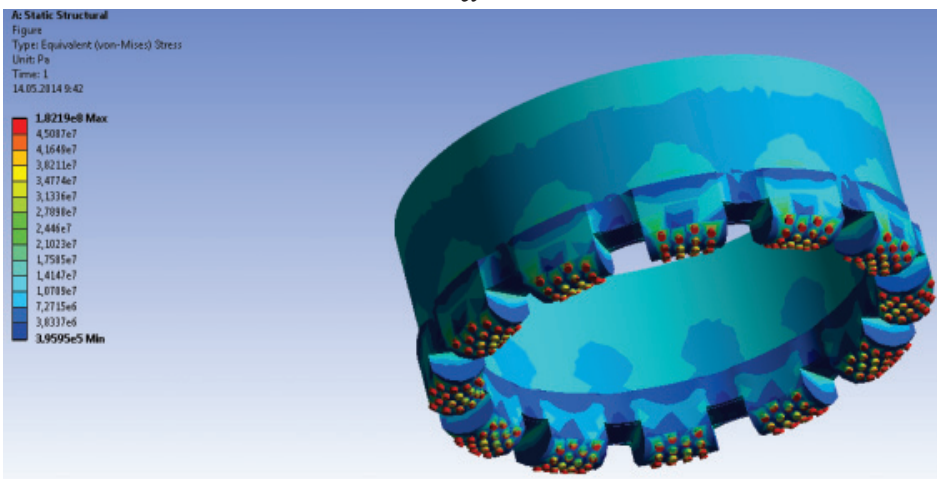

$b$

Pис. 5. Схема распределения механических напряжений: а) в одном максимально нагруженном алмазе при работе коронки; $b$ ) в коронке с учетом действующей осевой нагрузки и крутящего момента

Fig. 5. Scheme of mechanical stresses distribution: a) in one maximally loaded diamond while at crown operation; $b$ ) in the crown, taking into account the effective axial load and torque
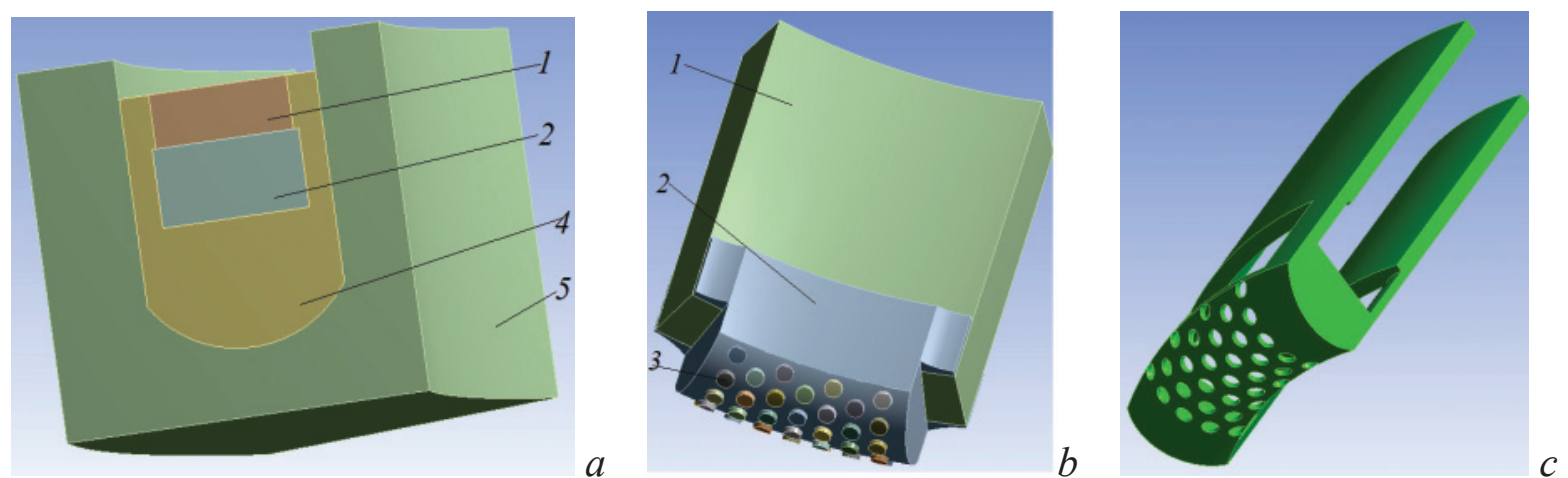

Puc. 6. Пример модели взаимодействия алмазной однослойной коронки с породой на забое скважины: а) модель в сборе; b) домен корпуса, сектора и алмазов; с) домен воды. 1 - корпус; 2 - сектор; 3 - алмаз; 4 - очистной агент; 5 - порода

Fig. 6. Example of a model of a diamond single-layer crown interaction with a rock at well bottom: a) assembly model; $b$ ) domain of corps, sector and diamonds; c) domain water. 1 is the body; 2 is the sector; 3 is the diamond; 4 is the cleaning agent; 5 is the rock

Для каждого домена задаются начальные граничные условия теплообмена. Сопряжение между доменами описывается законами сохранения энергии, заложенными в системе моделирования. Передача тепла от домена к домену происходит без потерь и сопротивлений. При этом учитывается относительное вращение доменов. Для вращающихся доменов и на контакте с ними задается граничное условие вращающейся поверхности с заданной постоянной частотой вращения. С целью прибли- 

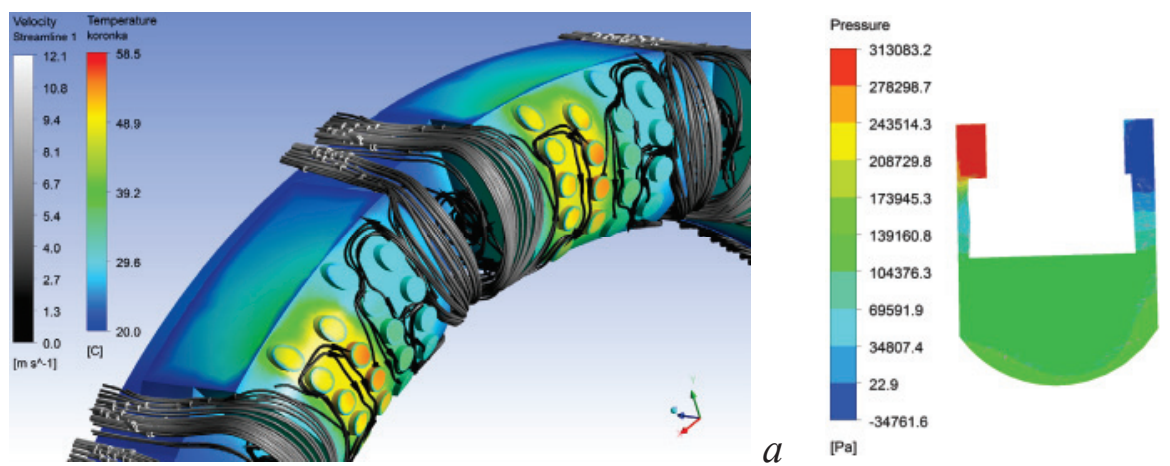

Рис. 7. Пример результатов моделирования: а) температура нагрева и скорость течения жидкости; b) распределение давления жидкости при бурении скважины коронкой

Fig. 7. Example of simulation results: a) heating temperature and fluid flow rate; b) fluid presser distribution during crown drilling

жения модели к реальным условиям на границах модели задается коэффициент теплопередачи соответствующей породы [26].

Составленная 3D модель позволяет определять температуру, скорость течения жидкости, давление в любой точке призабойной зоны. Примеры результатов моделирования приведены на рис. 7.

\section{Заключение}

Для разработки нового породоразрушающего инструмента, отвечающего современным требованиям, необходимо достоверное исследование его работы на забое и использование прогрессивных инновационных подходов [27]. Предложенный пример исследования работы алмазной коронки с целью проектирования оптимальной её конструкции показывает, что

\section{СПИСОК ЛИТЕРАТУРЫ}

1. Нескоромных В.В., Попова М.С. Основы системного подхода к проектированию бурового инструмента // Научно-технический журнал «Строительство нефтяных и газовых скважин на суше и на море». - 2018. - № 8. - С. 26-31.

2. Нескоромных В.В., Попова М.С. Разработка алмазного инструмента с применением данных компьютерного моделирования и результатов системных исследований // Научно-технический журнал «Инженер-нефтянник». - 2018. - № 3. C. $18-26$.

3. Нескоромных В.В. Разрушение горных пород при бурении скважин. - М.: ИНФРА-М; Красноярск: Сибирский федеральный университет, 2015. - 336 c.

4. Product Catalog. Smith Bits. A Schlumberger Company. 17-BDT310907 Copyright 2018 Schlumberger. All rights reserved. URL: https://www.slb.com/-/media/files/smith/catalogs/bits-catalog (дата обращения: 05.11.2018).

5. Интегрированные системы и технологии. Каталог компании Schlumberger. URL: https://sis.slb.ru/ (дата обращения: 05.11.2018).

6. Su 0., Ali Akcin. Numerical simulation of rock cutting using the discrete element method // International Journal of Rock Mechanics and Mining Sciences. - 2011. - № 48 (3). - P. 434-442.

7. Huang H., Lecampion B., Detournay E. Discrete element modeling of tool-rock interaction I: Rock cutting // International Journal for Numerical and Analytical Methods in Geomechanics. - 2013. - № 37 (13). - P. 1913-1929.
- бурение скважин как модель является сложной системой моделирования, поэтому научные исследования процессов бурения должны основываться на комплексном подходе [1];

- решение задач моделирования бурения возможно с применением современных средств программирования;

- компьютерное моделирование дает возможность учитывать все факторы, влияющие на результат бурения;

- компьютерное моделирование позволяет проводить исследования даже в мелких элементах и труднодоступных местах расчётной схемы;

- метод компьютерного моделирования облегчает процесс анализа и визуализации результатов исследования.

8. Integrated FEA modeling offers system approach to drillstring optimization / H. Aslaksen, M. Annand, R. Duncan, L. Paez, U. Tran et al. // SPE/IADC Drilling Conference, Proceedings. USA, Miami, 2006. - P. 669-684.

9. Александров А.Н., Кищенко М.А., Рогачев М.К. Моделирование образования твердых органических частиц в высокопарафинистой пластовой нефти // Научно-технический журнал «Инженер-нефтянник». - 2018. - № 2. - С. 42-49.

10. Hasan A.R., Kabir C.S. Wellbore heat-transfer modeling and applications // Journal of Petroleum Science and Engineering. 2012. - № 96-97. - P. 109-119.

11. Dong G., Chen P. 3D numerical simulation and experiment validation of dynamic damage characteristics of anisotropic shale for percussive-rotary drilling with a full-scale PDC bit // Energies. 2018. - № 11 (6). - P. 1285-1287.

12. Optimization model for polycrystalline diamond compact bits based on reverse design / Z. Ai, Y. Han, Y. Kuang, Y. Wang, M. Zhang // Advances in Mechanical Engineering. - 2018. № 10 (6). - P. 1-12.

13. Сериков Д.Ю., Гаффанов Р.Ф. Исследование напряженно-деформируемого состояния двух смежных центробежно-объемного-армированных зубьев вооружения шарошечного бурового инструмента // Строительство нефтяных и газовых скважин на суше и на море. - 2018. - № 9. - С. 42-47.

14. Нескоромных В.В., Борисов К.И. Аналитическое исследование процесса резания-скалывания горной породы долотом с резцами PDC // Известия Томского политехнического университета. - 2013. - Т. 323. - № 1. - С. 191-195. 
15. Brook B. Principles of diamond tool technology for sawing rock // International Journal of Rock Mechanics and Mining Sciences. 2002. - № 39 (1). - P. 41-58.

16. Teale $R$. The concept of specific energy in rock drilling // International Journal of Rock Mechanics and Mining Sciences. - 1965. № 2 (1). - P. 57-73.

17. Production of coarse-grained high-strength microgrits to be used in drilling tools / 0.A. Zanevskii, S.A. Ivakhnenko, G.D. Il'nitskaya, A.P. Zakora, R.K. Bogdanov, A.A. Karakozov, M.C. Popova // Journal of Superhard Materials. - 2015. - V. 37. - № 2. P. $85-96$.

18. Определение максимальной углубки алмазных резцов однослойных коронок с радиальной раскладкой / А.А. Каракозов, М.С. Попова, С.Н. Парфенюк, Р.К. Богданов, А.П. Закора // Породоразрушающий и металлообрабатывающий инструмент - техника и технология его изготовления и применения. Сборник научных трудов. - Киев: Институт сверхтвердых материалов им. В.Н. Бакуля Национальной академии наук Украины, 2012. - Вып. 15. - С. 203-206.

19. Исследование и разработка конструкции однослойной буровой коронки с синтетическими монокристаллами алмаза / А.А. Каракозов, М.С. Попова, С.Н. Парфенюк, Р.К. Богданов, А.П. Закора // Породоразрушающий и металлообрабатывающий инструмент - техника и технология его изготовления и применения. Сборник научных трудов. - Киев: Институт сверхтвердых материалов им. В.Н. Бакуля национальной академии наук Украины, 2014. - Вып. 17. - С. 73-79.
20. Буровой инструмент для геологоразведочных скважин: справочник / Н.И. Корнилов, Н.Н. Бухарев, А.Т. Киселев и др. / под ред. Н.И. Корнилова. - М.: Недра, 1990. - 395 с.

21. Королев А.Л. Компьютерное моделирование. - М.: БИНоМ. J3, 2013. -230 c.

22. Кринг Т.Дж. LabVIEW для всех. 4-е изд., переработанное и дополненное. - М.: ДМК Пресс, 2011. - 904 с.

23. Тупицына А.И. Методы компьютерного моделирования физических процессов и сложных систем. - СПб.: Университет информационных технологий, механики и оптики, 2014. -48 с.

24. Магда Ю.C. LabVIEW: практический курс для инженеров и разработчиков. - М.: ДМК Пресс, 2012, 208 с.

25. Васильев А.С., Лашманов 0.Ю. Основы программирования в среде LabVIEW. - СПб.: Университет информационных технологий, механики и оптики, 2015. - 82 с.

26. Исследование теплового режима однослойной алмазной коронкой с синтетическими монокристаллами / А.А. Каракозов, М.С. Попова, С.Н. Парфенюк, Р.К. Богданов, А.П. Закора // Наукові праці Донецького національного технічного університету. Серія: Гірничо-геологічна. - 2015. - № 1 (22). - С. 39-44.

27. Инновационные подходы к конструированию высокоэффективного породоразрушающего инструмента / А.Я. Третьяк, В.В. Попов, А.Н. Гроссу, К.А. Борисов // Горный информационно-аналитический бюллетень. - 2017. - № 8. C. $225-230$.

Поступила: 12.11 .2018 г.

\section{Информация об авторах}

Попова M.С., старший преподаватель кафедры технологии и техники разведки Института горного дела, геологии и геотехнологий Сибирского федерального университета.

Харитонов А.Ю., кандидат технических наук, сертифицированный LabVIEW-разработчик. 
UDC 622.24.051.64

\title{
COMPUTER SIMULATION OF PROCESSES AT WELL DRILLING
}

\author{
Marina S. Popova', \\ alleniram83@mail.ru \\ Anton Yu. Kharitonov ${ }^{2}$, \\ ant.kharitonov@gmail.com \\ 1 Siberian Federal University, \\ 95, «Krasnoyarskiy rabochy» newspaper avenue, Krasnoyarsk, 660095, Russia. \\ 2 LabVIEW freelancer, \\ Moiveien 2, 4460, Moi, Norway.
}

Relevance. The main drilling development direction is improving technical and economic indicators. The main direction is the new rockcutting tools generation, which ensures the effective rock destruction. To improve the technical and economic drilling indicators it is necessary to study the processes occurring during well drilling. Often the processes are complex and need to be detailed. This applies to narrow, hard-to-reach places in the bottomhole zone. In some cases, experimental research methods are difficult, expensive, or completely unavailable. In addition, the main modern requirement for scientific research is an integrated approach. It consists in a comprehensive and simultaneous consideration of all processes occurring during well drilling. In this regard, a relevant method is the computer simulation. This method studying drilling accurately, visually and reliably.

The aim of the research is the computer simulation of the processes occurring during drilling.

Objects: processes occurring during well drilling.

Methods: simulation modeling, analytical studies, analysis.

Results. The authors have identified the main drilling processes that need to be explored in the design and development of a new generation rock cutting tools. The paper considers the possibilities of computer modeling in study of drilling with diamond crowns. On the simplified model example for the interaction of rock cutting tools with rocks, software products are given. The results of their use are shown: the thickness of a rock layer removed by any diamond crowns, the tool location coordinates at any time, the heating temperature of the diamond single-layer crown, the distribution of mechanical stresses. The authors compared the modeling of the same drilling processes using different software products. The features, modeling accuracy of drilling processes are revealed. The computer simulation of drilling processes is grounded. The paper demonstrates the advantages of computer simulation over the experiment.

\section{Key words:}

Computer modelling, drilling, rock cutting tool, programming, parametric model, diamond layout, drill bit.

\section{REFERENCES}

1. Neskoromnykh V.V., Popova M.S. Basis of the system approach to drilling tool design. Construction of oil and gas wells on land and sea, 2018, no. 8, pp. 26-31. In Rus.

2. Neskoromnykh V.V., Popova M.S. Diamond tool development using computer modelling data and system reserch results. Petroleum Engineer, 2018, no. 3, pp. 18-26. In Rus.

3. Neskoromnykh V.V. Razrushenie gornykh porod pri burenii skvazhin [Destruction of rocks during drilling]. Moscow, INFRA-M Publ.; Krasnoyarsk, SFU Publ., 2015. 336 p.

4. Product Catalog. Smith Bits. A Schlumberger Company. 17-BDT310907 Copyright 2018 Schlumberger. All rights reserved. Available at: https://www.slb.com/-/media/files/smith/cata$\operatorname{logs} /$ bits-catalog (accessed 5 November 2018).

5. Integrated systems and technologies. Catalog of the Company Schlumberger. Available at: https://sis.slb.ru/ (accessed 5 November 2018).

6. Su 0., Ali Akcin. Numerucal simulation of rock cutting using the discrete element method. International Journal of Rock Mechanics and Mining Sciences, 2011, no. 48 (3), pp. 434-442.

7. Huang H., Lecampion B., Detournay E. Discrete element modeling of tool-rock interaction I: Rock cutting. International Jour nal for Numerical and Analytical Methods in Geomechanics, 2013, no. 37 (13), pp. 1913-1929.

8. Aslaksen H., Annand M., Duncan R., Paez L., Tran U. Integrated FEA modeling offers system approach to drillstring optimization. SPE/IADC Drilling Conference. Proceedings. USA, Miami, 2006, 2006. pp. 669-684.
9. Aleksandrov A.N., Kishchenko M.A., Rogachev M.K. Modeling the formation of solid organic particles in highly paraffin reservoir oil. Petroleum Engineer, 2018, no. 2, pp. 42-49. In Rus.

10. Hasan A.R., Kabir C.S. Wellbore heat-transfer modeling and applications. Journal of Petroleum Science and Engineering, 2012, no. 96-97, pp. 109-119.

11. Dong G., Chen P. 3D numerical simulation and experiment validation of dynamic damage characteristics of anisotropic shale for percussive-rotary drilling with a full-scale PDC bit. Energies, 2018, no. 11 (6), pp. 1285-1287.

12. Ai Z., Han Y., Kuang Y., Wang Y., Zhang M. Optimization model for polycrystalline diamond compact bits based on reverse design. Advances in Mechanical Engineering, 2018, no. 10 (6), pp. 1-12.

13. Serikov D.Yu., Gaffanov R.F. Investigation of the stress-strain state of two adjacent centrifugal-volume-reinforced teeth of the armament of a bit drilling tool. Construction of oil and gas wells on land and sea, 2018, no. 9, pp. 42-47. In Rus.

14. Neskoromnykh V.V., Borisov K.I. Analytical study of rock cutting-spallation with PDC cutters chisels. Bulletin of the Tomsk Polytechnic University, 2013, vol. 323, no. 1, pp. 191-195.

15. Brook B. Principles of diamond tool technology for sawing rock. International Journal of Rock Mechanics and Mining Sciences, 2002, no. 39 (1), pp. 41-58.

16. Teale $\mathrm{R}$. The concept of specific energy in rock drilling. International Journal of Rock Mechanics and Mining Sciences, 1965, no. 2 (1), pp. 57-73.

17. Zanevskii O.A., Ivakhnenko S.A., Il'nitskaya G.D., Zakora A.P., Bogdanov R.K., Karakozov A.A., Popova M.C. Production of co- 
arse-grained high-strength microgrits to be used in drilling tools. Journal of Superhard Materials, 2015, vol. 37, no. 2, pp. 85-96.

18. Karakozov A.A., Popova M.S., Parfenuk S.N., Bogdanov R.K. Zakora A.P. Opredelenie maksimalnoy uglubki almaznykh reztsov odnosloynykh koronok s radialnoy raskladkoy [Determination of the maximum deepening of diamond incisors of single-layered crowns with a radial layout]. Porodorazrushayushchiy $i$ metalloobrabatyvayushchiy instrument - tekhnika i tekhnologiya ego izgotovleniya i primeneniya. Sbornik nauchnykh trudov [Rock cutting and metalworking tools - machinery and technology of its production and application. Collection of scientific papers]. Kiev, Institute of Superhard Materials Publ., 2012. Iss.15, pp. 203-206.

19. Karakozov A.A., Popova M.S., Parfenuk S.N., Bogdanov R.K., Zakora A.P. Issledovanie i razrabotka konstruktsii odnosloynoy burovoy koronki s sinteticheskimi monokristallami almaza [Research and development of the contraction of a single-layer drill bit with synthetic diamond single crystals]. Porodorazrushayushchiy $i$ metalloobrabatyvayushchiy instrument - tekhnika $i$ tekhnologiya ego izgotovleniya i primeneniya. Sbornik nauchnykh trudov [Rock cutting and metalworking tools - machinery and technology of its production and application. Collection of scientific papers]. Kiev, ISM Publ., 2014. Iss.17, pp. 73-79.

20. Kornilov N.I., Bukharev N., Kiselev A.T. Burovoy instrument dlya geologorazvedochnykh skvazhin: spravochnik [Drilling tools for exploration wells: reference]. Moscow, Nedra Publ., 1990. $395 \mathrm{p}$.

\section{Information about the authors}

Marina S. Popova, senior teacher, Siberian Federal University.

Anton Yu. Kharitonov, Cand. Sc., certified LabVIEW developer.
21. Korolev A.L. Kompyuternoe modelirovanie [Computer modelling]. Moscow, BINOM Publ, 2013. 230 p.

22. Kring T.J. LabVIEW dlya vsekh [LabVIEW for everyone]. $4^{\text {th }}$ ed. Moscow, DMK Press, 2011. 904 p.

23. Tupitsyna A.I. Metody kompyuternogo modelirovaniya fizicheskikh protsessov i slozhnykh sistem [Methods of computer modeling of physical processes and complex systems]. St. Petersburg, University of Information Technologies, Mechanics and Optics Publ., 2014. 48 p.

24. Magda Yu.S. LabVIEW: prakticheskiy kurs dlya inzhenerov i razrabotchikov [LabVIEW: a practical course for engineers and developers]. Moscow, DMK Press, 2012. 208 p.

25. Vasiliev A.S., Lashmanov 0.Yu. Osnovy programmirovaniya $v$ srede LabVIEW [Basics of programming in the LabVIEW]. St-Petersburg, University of Information Technologies, Mechanics and Optics Publ., 2015. 82 p.

26. Karakozov A.A., Popova M.S., Parfenuk S.N., Bogdanov R.K., Zakora A.P. Study of the thermal regime of a single-layer diamond crown with synthetic single crystals. Scientific works of Donetsk National Technical University. Series: Mining and geological, 2015, no. 1 (22), pp. 39-44. In Rus.

27. Tretyak A.Ya., Popov V.V., Grossu A.N., Borisov K.A. Innovative approaches to the design of highly efficient rock cutting tools. Mining Information and Analytical Bulletin, 2017, no. 8, pp. 225-230. In Rus. 